\title{
COMMENT
}

\section{IL-17A moonlighting in lung type 2 immunity}

\author{
Roland Ruscher ${ }^{1}$ and Paul R. Giacomin (D) \\ Mucosal Immunology (2020) 13:849-851; https://doi.org/10.1038/s41385-020-0329-z
}

Both Type 2 inflammation and the cytokine IL-17A are implicated in allergic asthma, yet how these distinct immune responses are interlinked in the lung remains poorly understood. Utilizing helminth infection models in mice to induce pulmonary Type 2 immunity, Ajendra et al. reveal a molecular switch, where IL-17A initially acts to promote the establishment of the Type 2 response to infection by inhibiting IFN $\gamma$ production, but at later stages IL$17 \mathrm{~A}$ acts to reduce Type 2 responses, protecting the lung tissue from detrimental chronic inflammation. Interestingly, the multitasking function of IL-17A in regulating lung Type 2 inflammation was even observed when mice were infected with a different helminth species that resides entirely in the gastrointestinal tract. These novel findings advance our understanding of how IL-17A plays multiple "moonlighting" functions in regulating pulmonary Type 2 inflammation, which has implications for the etiology of severe asthma.

Type 2 immune responses play critical roles in immunity to helminths, but also in allergic conditions. A different branch of the immune system, involving IL-17A, is critical in protection against bacterial and fungal infections. Emerging evidence suggests that these two facets of the immune response are intricately linked in conditions such as allergic asthma, where Type 2 responses and IL-17A are both associated with inflammation and lung tissue damage. However, the mechanisms of interplay between Type 2 and IL-17A responses are incompletely understood.

In this issue of Mucosal Immunology, Ajendra et al. reveal previously unrecognized ways that IL-17A "multi-tasks" in the lung, to both promote and inhibit Type 2 inflammation, depending on timing and the surrounding inflammatory milieu, using the Nippostrongylus brasiliensis helminth infection model in mice. ${ }^{1} N$. brasiliensis is a hookworm-like parasite that after infecting its host through the skin, migrates quickly through the lung between days 1 and 3 post-infection inducing damage and inflammation, before reaching its final destination, the intestines by days 5-7. The authors show that in this setting, IL-17A expression is rapidly induced in $\gamma \delta$ T cells in the lung at day 2, which acts to suppress the production of IFNY by T cells and NK cells, facilitating the induction of a type 2 response while the helminth passes through the lung. The absence of IL-17A in knockout mice resulted in impaired Type 2 responses such as CD4 ${ }^{+}$T-helper type 2 (Th2) cells, group 2 innate lymphoid cells (ILC2s) and neutrophils, and increased rates of parasite migration to the gut. In contrast, later in the course of infection while the parasites were residing in the small intestine (day 6), IL-17A displayed an inhibitory effect on the Type 2 inflammatory response in the lung, in particular the lung ILC2 response. An overview is shown in Fig. 1. Intriguingly, while CD4 ${ }^{+}$Th2 cells were increased in the lung in presence of IL-17A in the early stages of infection, $\mathrm{CD}^{+}{ }^{+} \mathrm{Th} 2$ cell numbers were not altered in the lung- draining lymph nodes, indicating that the underlying mechanism(s) may be tissue-specific. Further tissue-specific dual functions of IL17A were evidenced when the authors employed the Trichuris muris model of helminth infection, which exclusively occupies the gut and does not pass through the lungs. In this model, IL-17A-dependent induction of Type 2 immunity was still observed in the pulmonary tract yet not in the intestines. Together, these findings expand the mechanistic understanding of how IL-17A can under certain contexts play roles in promoting Type 2 responses, but have a "moonlighting" function to suppress those same responses as a tissue-specific negative feedback mechanism.

Previous studies by the same research group had identified IL$17 \mathrm{~A}$ as a promoter of neutrophil-associated Type 2 inflammation and tissue damage in the lung in the same helminth model, where chitinase-like proteins (CLPs) such as Ym1 and BRP-39 were shown to promote IL-17A production by $\gamma \delta \mathrm{T}$ cells. ${ }^{2}$ The present study addresses the gap in the knowledge regarding how IL-17A promotes Type 2 responses, by implicating suppression of IFNY, and extends these findings beyond the lung tissue. While IL-17A and IFN $\gamma$ had been linked previously, their interdependent dynamics in the context of pulmonary Type 2 inflammation were poorly understood. Despite these new findings, some important questions still remain regarding interplay between IL-17A, IFNy and Type 2 responses in the lung, and other tissues. It remains unclear how IL-17A acts to suppress lung Type 2 responses later in the infection, which may involve an IFN $\gamma$-independent mechanism. In addition, while the main source of IL-17A following lung $N$. brasiliensis infection was $\gamma \delta$ $T$ cells, questions still remain regarding the sources of IL-17A in the setting of intestinal $T$. muris infection, and what actually triggers its expression. It is possible that lung-resident $\gamma \delta$ T cells and/or distinct IL-17A producing cells may respond to circulating factors such as CLPs induced by the presence of the parasites in the gut, similar to the scenario with lung $N$. brasiliensis infection. Alternatively, IL-17A-producing cells may migrate to the lung from other tissues such as the gut. Lastly, Ajendra et al. used a high dose of T. muris infection (200 parasite eggs), while previous studies employing lower doses of $30 \mathrm{~T}$. muris eggs reported increased IFN $\gamma$ responses in the lung, which counteracted papain-induced Type 2 airway inflammation. ${ }^{3}$ This highlights that the intensity of infection may influence the systemic immune responses elicited by gastrointestinal helminths. A more complete understanding of these matters may be required to discern how targeting the IL-17IFNY-Type 2 axis may be a rational therapeutic approach for asthma or helminth infections.

These cumulative findings regarding IL-17A have expanded on previous knowledge that pulmonary Type 2 responses can be regulated by a network of cell types, cytokines, and other molecules.

${ }^{1}$ Centre for Molecular Therapeutics, The Australian Institute of Tropical Health and Medicine, James Cook University, Cairns, QLD, Australia

Correspondence: Paul R. Giacomin (paul.giacomin@jcu.edu.au)

Received: 29 June 2020 Accepted: 6 July 2020

Published online: 29 July 2020 


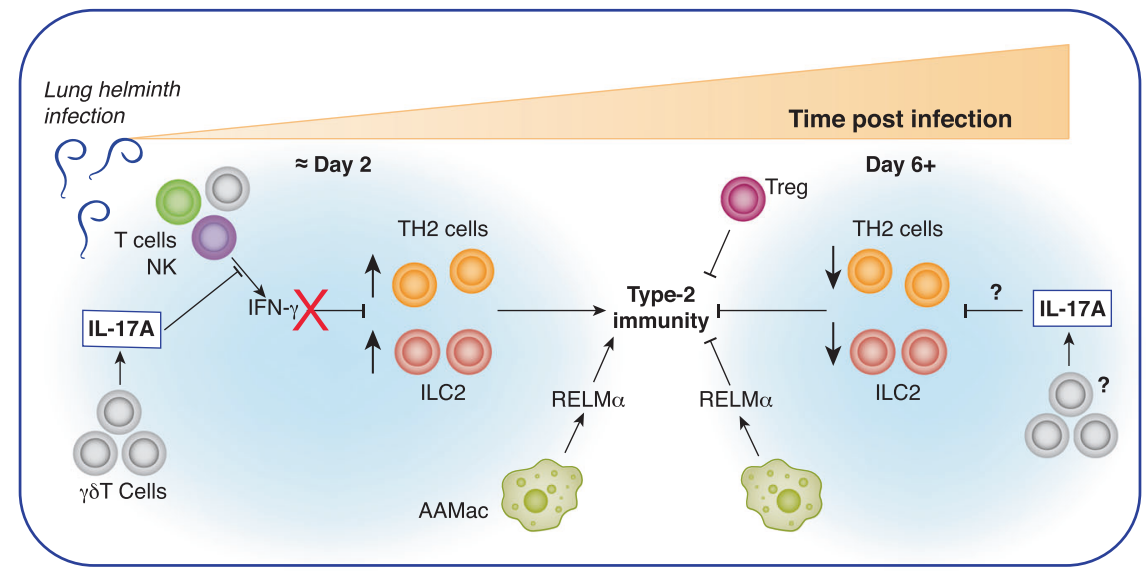

Fig. 1 Pulmonary responses to helminth infections. Under homeostatic conditions, T cell and natural killer (NK) cell derived IFN $\gamma$ keeps Type 2 immunity in check by suppressing Th2 cell and ILC2 responses. However, in the early stages (day 2) following infection with N. brasiliensis, IL-17A secreted by $\gamma \delta$ T cells inhibits IFN $\gamma$ production, thereby facilitating initiation of a Type 2 response. During later infection (day 6 ), IL-17A acts instead to suppress this Type 2 response, preventing excessive inflammation and tissue damage. ${ }^{1}$ The source of IL-17A and the mechanism of suppression of Type 2 immunity remain unclear. This IL-17A-dependent molecular switch adds to previously characterized mechanisms of how Type 2 immunity in the lung is regulated during helminth infections, including the similarly multitasking factor RELM $\alpha$, produced by alternatively activated macrophages (AAMac), which can both promote early Type 2 responses to helminths, ${ }^{4}$ and act to suppress these responses at later times. ${ }^{5,6}$ Similarly, induction of regulatory T-cell (Treg) responses by helminths is associated with suppression of pulmonary Type 2 responses, which can be effective at limiting house dust mite-induced asthma, ${ }^{7}$ and limit Th2 cell-dependent protective immunity 8 .

For example, alternatively activated macrophage-derived resistin-like molecule (RELM)a is another multitasking factor that plays positive roles in Type 2 responses to helminth infection, by inducing antiparasitic responses, ${ }^{4}$ but in other contexts act to suppress pulmonary Type 2 responses to protect against detrimental lung inflammation (Fig. 1). ${ }^{5,6}$ Induction of regulatory T cells (Treg) is also a feature of helminth infections, acting simultaneously with, and often counteracting Type 2 responses. For example, infection with the gastrointestinal helminth Heligmosomoides polygyrus attenuates allergic Type 2 responses in the lung via changes in the microbiome and short-chained fatty acid-mediated enhancement in Treg function, ${ }^{7}$ while secondary $N$. brasiliensis infection elicits IL-33responsive Tregs that can suppress memory Th2 cell function. ${ }^{8}$ Hence, it is apparent that there are multiple distinct mechanisms by which the immune system tightly controls both the induction and resolution of Type 2 responses in the lung, and pleiotropic cytokines like IL-17A and RELMa are central for this.

While the results in the current Ajendra et al. study indicate that initiation of Type 2 responses in the lung by IL-17A was lung sitespecific, intestinal Type 2 immunity in T. muris infected mice was previously also found to be facilitated by IFNy suppression, mediated by thymic stromal lymphopoietin (TSLP). ${ }^{9}$ It is therefore conceivable that complementary mechanisms to regulate Type 2 immunity through IFN $\gamma$ suppression in different tissues may be mediated by distinct regulatory factors such as IL-17A and TSLP. There are several potential consequences of increased IL-17A expression and subsequent IFNy suppression following helminth infection or during allergic disease that could be explored further. For example, IFNy plays important roles in the clearance of viral or bacterial infections that affect the pulmonary tract, such as influenza or tuberculosis. Recently, reduced IFNy expression, especially by $\mathrm{CD}^{+} \mathrm{T}$ cells, was associated with severe acute respiratory syndrome coronavirus 2 (SARS-CoV-2/COVID-19). ${ }^{10}$ Helminth-driven suppression of IFNY by IL-17A could therefore be detrimental in the context of viral infections. On the other hand, induction of pulmonary IL-17A responses by lung and gutdwelling helminths may also influence immunity to extracellular bacterial and fungal infection, or affect the composition of the lung microbiome. These possible scenarios additionally highlight the imperative to further investigate the details of the
parasite-IL-17A-IFNy axis, including future studies in human health and disease.

Overall, Ajendra et al. ${ }^{1}$ presents an exciting new model in which helminth infection-driven IL-17A is central for both the initiation of a Type 2 response, and its subsequent inhibition to help prevent chronic Type 2 inflammation and tissue damage. Suppression of IFNy expression in the lung appears to be a critical intermediate event, and appears to be a tissue-specific phenomenon. A better understanding of the mechanisms of how IL-17A has multiple "moonlighting" jobs in regulating Type 2 mediated pulmonary inflammation could in the future set the stage for novel interventions in diseases such as severe allergic asthma, but also the development of anti-helminth vaccines that target lung stages of parasites.

\section{ACKNOWLEDGEMENTS}

This study was supported by Australian National Health and Medical Research Council (NHMRC) project grant (P.R.G).

\section{AUTHOR CONTRIBUTIONS}

R.R and P.R.G wrote original draft and approved the final paper.

\section{ADDITIONAL INFORMATION}

Competing interests: The authors declare no competing interests.

\section{REFERENCES}

1. Ajendra A., et al. IL-17A both initiates, via IFNy suppression, and limits the pulmonary type-2 immune response to nematode infection. Mucosal. Immunol. (2020). https://doi.org/10.1038/s41385-020-0318-2.

2. Sutherland, T. E. et al. Chitinase-like proteins promote IL-17-mediated neutrophilia in a tradeoff between nematode killing and host damage. Nat. Immunol. 15, 1116-1125 (2014).

3. Chenery, A. L. et al. Low-dose intestinal trichuris muris infection alters the lung immune microenvironment and can suppress allergic airway inflammation. Infect. Immun. 84, 491-501 (2016).

4. Krljanac, B. et al. RELMa-expressing macrophages protect against fatal lung damage and reduce parasite burden during helminth infection. Sci. Immunol. 4, eaau3814 (2019). 
5. Nair, M. G. et al. Alternatively activated macrophage-derived RELM-\{alpha\} is a negative regulator of type 2 inflammation in the lung. J. Exp. Med. 206, 937-952 (2009).

6. Pesce, J. T. et al. Retnla (relmalpha/fizz1) suppresses helminth-induced Th2-type immunity. PLoS Pathog. 5, e1000393 (2009).

7. Zaiss, M. M. et al. The intestinal microbiota contributes to the ability of helminths to modulate allergic inflammation. Immunity 43, 998-1010 (2015).
8. Obata-Ninomiya, K. et al. CXCR6(+)ST2(+) memory T helper 2 cells induced the expression of major basic protein in eosinophils to reduce the fecundity of helminth. Proc. Natl Acad. Sci. USA 115, E9849-e58 (2018).

9. Taylor, B. C. et al. TSLP regulates intestinal immunity and inflammation in mouse models of helminth infection and colitis. J. Exp. Med. 206, 655-667 (2009).

10. Chen, G. et al. Clinical and immunological features of severe and moderate coronavirus disease 2019. J. Clin. Investig. 130, 2620-2629 (2020). 